Portland State University

PDXScholar

\title{
Quantifying and Enforcing Two-dimensional Symmetries in Scanning Probe Microscopy Images
}

\author{
Peter Moeck \\ Portland State University, pmoeck@pdx.edu \\ Marius Toader \\ Chemnitz University of Technology \\ Mahmoud Abdel-Hafiez \\ Chemnitz University of Technology \\ Michael Hietschold \\ Chemnitz University of Technology
}

Follow this and additional works at: https://pdxscholar.library.pdx.edu/phy_fac

Part of the Physics Commons

Let us know how access to this document benefits you.

\section{Citation Details}

Moeck, P., Toader, M., Abdel-Hafiez, M., \& Hietschold, M. (2009, September). Quantifying and enforcing two-dimensional symmetries in scanning probe microscopy images. In Frontiers of Characterization and Metrology for Nanoelectronics: 2009 (Vol. 1173, No. 1, pp. 294-298). AIP Publishing.

This Article is brought to you for free and open access. It has been accepted for inclusion in Physics Faculty Publications and Presentations by an authorized administrator of PDXScholar. Please contact us if we can make this document more accessible: pdxscholar@pdx.edu. 


\title{
Quantifying and enforcing two-dimensional symmetries in scanning probe microscopy images
}

\author{
Peter Moeck $^{1}$, Marius Toader ${ }^{2}$, Mahmoud Abdel-Hafiez $^{2}$, and Michael Hietschold ${ }^{2}$ \\ ${ }^{1}$ Nano-Crystallography Group, Department of Physics, Portland State University, Portland, OR 97207-0751, U.S.A. \\ \& Oregon Nanoscience and Microtechnologies Institute, www.onami.us \\ ${ }^{2}$ Solid Surfaces Analysis Group \& Electron Microscopy Laboratory, Institute of Physics, Chemnitz University of \\ Technology, D-09126 Chemnitz, Reichenhainer Straße 70, Federal Republic of Germany
}

\begin{abstract}
The overall performance and correctness of the calibration of all kinds of traditional scanning probe microscopes can be assessed in a fully quantitative way by means of "crystallographic" processing of their twodimensional (2D) images from samples with 2D periodic (and preferably highly symmetric) features. This is because crystallographic image processing results in two residual indices that quantify by how much the symmetry in a corresponding scanning probe microscopy image deviates from the symmetries of the possible plane groups of the periodic features of the sample. When a most probable plane symmetry group has been identified on the basis of crystallographic image processing, the symmetry in the scanning probe microscopy image can be "enforced" in order to obtain "clearer" images, effectively removing the less than ideal "influence" of the microscope on the imaging processes. This paper illustrated the crystallographic image processing procedure for scanning tunneling microscopy images that were recorded from a monolayer of a phthalocyanine on two different types of substrates.
\end{abstract}

Keywords: scanning tunneling microscopy, scanning probe microscopy, two-dimensional symmetry

PACS: 68.37.- d, 68.37.Ef, 68.37.Ps, 68.55.-a, 68.55.at, 68.60.Wm, 68.65.-k, 68.90.+g, 07.79.-v

\section{INTRODUCTION}

Many different kinds of scanning probe microscopes (SPMs) have been developed since the inventions of the scanning tunneling microscope (STM) and the atomic force microscope (AFM) in the early and mid 1980s. The defining features of this type of microscope are a very fine probe that is scanned laterally in two dimensions (2D), in very fine steps, very close to the surface of a sample (while being controlled by a feedback mechanism) and a probesample interaction signal that is recorded at each scanning increment.

This signal can then be digitized and prepared for its displays as function of the magnified scanning steps. Between the scanning steps, the signal may be interpolated to provide for a smooth display. The digitized signal can, for example, be converted by a certain code into colors for a pseudo three-dimensional perspective false-color display of the probe-sample interactions. Traditional SPMs produce only single valued surface topography and probe-sample interaction maps so that the shapes of the imaged objects are only a subset of all possible threedimensional shapes.

To a reasonably good approximation, a 2D-image of the probe-sample interactions as recorded in a traditional SPM can be obtained by simply ascribing the digitized signal directly to its respective scanning increments in 2D. This image corresponds then to an orthogonal projection of the signal from the third dimension (z-direction) of the sample on to the 2D scanning ( $\mathrm{x}-\mathrm{y}$ plane) plane. (Note that while this is strictly true only for pure constant-tunneling-current images, it is a rather good approximation for images that were taken with most traditional SPMs.)

Like any other 2D image, these images can be subjected to image processing routines in order to quantify the information contained in them. A proper calibration of the whole microscope is essential for the quantification of the information. The calibration of a SPM can be assessed by the use of calibration standards [1], and may involve deconvolution of the scanning probe tip geometry (so called "tip dilation") in order to recover the sample surface geometry [2].

A very popular form of image processing is a kind of "2D background correction" on the basis of prior knowledge of the expected strength of the probesample interaction at different lateral positions of the sample. For example, if one expects the probe-sample interaction strength to be the same at the margins of opposite edges of the image, one may define a plane by these edges and enforce this plane as the new x-y plane of the image. This procedure is commonly referred to as "removal of sample tilt" or "z-offset removal"

CP1173, Frontiers of Characterization and Metrology for Nanoelectronics: 2009

edited by D. G. Seiler, A. C. Diebold, R. McDonald, C. M. Garner, D. Herr, R. P. Khosla, and E. M. Secula 2009 American Institute of Physics 978-0-7354-0712-1/09/\$25.00 
because different probe-sample interaction strengths at the margins of a sample may be simply due to the sample plane being not perpendicular to the scanning probe.

For SPM images from 2D periodic objects, crystallographic image processing (CIP) [3] can be utilized to quantify the 2D symmetry [4] in the images. Combined usage of calibration standards (with known periodic features and preferably high plane symmetry) and CIP allows for a quantitative assessment of the overall performance and correctness of the calibration of a SPM. This will be dealt with at the end of this paper after a discussion of the basics of CIP and its application to STM images of a fluorinated transitionmetal phthalocyanine monolayer on a noble metal and on graphite.

\section{CRYSTALLOGRAPHIC IMAGE PROCESSING}

CIP is widely employed in electron crystallography to aid the extraction of structure factors from highresolution phase contrast images that are recorded with transmission electron microscopes [5,6]. The underlying 2D symmetry quantification principles of CIP are, however, completely general. This is because there are just 17 symmetry groups for periodic objects in 2D [7]. (To aid the discerning of the orientation of mirror and glide lines, 21 plane group symbols [7] are used in this paper and popular CIP software [3]).

The amplitude (F) and phase (a) of the Fourier coefficients of the intensity of any image have to obey certain symmetry relations and restrictions in order to belong to one of these plane groups [6]. The amplitude and phase residuals of the Fourier coefficients of a 2Dimage will be different for each of the plane groups and provide, therefore, a means to quantify symmetry in 2D. For an exact adherence of an image to one of the 16 plane groups that possess more point symmetry elements than the identity element (which is of course an element of all 17 groups), these residuals would be zero. This would correspond to a measurement without any systematic and random errors.

The amplitude $\left(F_{\text {res }}\right)$ and phase $\left(a_{\text {res }}\right)$ residuals are defined by the relations [6]:

$$
\begin{aligned}
F_{\text {res }} & =\frac{\sum_{H K}\left|F_{\text {obs }}(H, K)\right|-\left|F_{\text {sym }}(H, K)\right|}{\sum_{H K}\left|F_{\text {obs }}(H, K)\right|} \text { in percentage (1) and } \\
\alpha_{\text {res }}= & \frac{\sum_{H K} w(H, K) \cdot\left|\alpha_{o b s}(H, K)-\alpha_{s y m}(H, K)\right|}{\sum_{H K} w(H, K)} \text { in degrees (2) }
\end{aligned}
$$

where the subscripts stand for observed and symmetrized, $w$ is a relative weight, and the sums are taken over all Fourier transform coefficient labels $H$ and $K$.

These residuals are trivial (in other words: meaningless as they are per definition zero) for plane group p1 and the application of the CIP procedure results in that special case only in a translation averaging over the periodic motive in the image. Since certain plane groups only exist for certain 2D lattices [7], it makes sense to determine the type of the lattice first by CIP and then to calculate the amplitude and phase residuals only for the respective plane groups.

\section{QUANTIFYING AND ENFORCING PLANE SYMMETRIES ON STM IMAGES OF F 16 CoPc MONOLAYERS}

STM images have been recorded from monolayers of fluorinated cobalt phthalocyanine $\left(\mathrm{F}_{16} \mathrm{CoPc}\right)$ on highly (0001) oriented pyrolytic graphite (technically knows as HOPG) and (110) oriented silver. Graphite possesses space group $\mathrm{P}_{3} \mathrm{mc}$ (No. 186) and the plane group of its (0001) surface is p6mm. Silver [8] and gold [9] (both possessing space group Fm $\overline{3}$ m, No. 225) with an (111) surface are also popular substrates with the p6mm symmetry for mono- and multi-layers of tin phthalocyanine ( $\mathrm{SnPc})$ [8] and cobalt phthalocyanine (CoPc) [9]. Other popular (0001) oriented substrates with the same plane group for STM analyses of phthalocyanines are $\mathrm{MoS}_{2}$ [10] and $\mathrm{WSe}_{2}$ (as both possess the space group $\mathrm{P}_{3} / \mathrm{mmc}$, No. 194). The silver (110) surface possesses plane group c2mm.

High-resolution transmission electron microscopy (HRTEM) studies, on the other hand, prefer (001) oriented $\mathrm{KCl}$ (which possesses plane group $\mathrm{p} 4 \mathrm{~mm}$, space group No. 225) [11] as substrate, because it can be readily removed from the deposit by dissolving in water. The $\mathrm{F}_{16} \mathrm{CoPc}$ molecule, Fig. 1 left hand side, and all homologous phthalocyanines possess in their planar projection the 2D point symmetry group $4 \mathrm{~mm}$.

Following Curie's symmetry principle [12], (but disregarding the differences in the sizes of the 2D unit cells and the underlying atomic near-surface structure, as well as possible preparation induced reconstructions,) one would expect from the possible intersections of symmetry elements that the epitaxial arrangement of an $\mathrm{F}_{16} \mathrm{CoPc}$ monolayer on strongly interacting "p6mm or c2mm substrates" should be either $\mathrm{c} 2 \mathrm{~mm}, \mathrm{p} 2 \mathrm{~mm}, \mathrm{p} 2, \mathrm{~cm}$, or pm. The inter-molecule interactions should be comparatively weak for these predictions to be borne out in experiments.

Free energy minimization between the deposit and substrates may result in higher or lower plane symmetries for these monolayers depending on the temperature. If the interaction energy between the deposit and the substrate is low in comparison to the energy of inter-molecule interactions, the monolayer may possess plane symmetries as high as $\mathrm{p} 4 \mathrm{~mm}$ or $\mathrm{p} 4$, i.e. groups that contain the four-fold axis of the point group of the molecule. This could be the case for a monolayer of $\mathrm{F}_{16} \mathrm{CoPc}$ on graphite, where it is not 
inconceivable that a highly symmetric " $2 \mathrm{D}$ monolayer crystal" may be observed under suitable conditions.

Stronger interactions between the substrate and the $\mathrm{F}_{16} \mathrm{CoPc}$ molecules are, on the other hand, to be expected for metallic substrates. As a consequence, we expect the plane symmetry of an $\mathrm{F}_{16} \mathrm{CoPc}$ monolayer to be much lower on silver (110) than on graphite. (Note also that the plane symmetry group of the silver (110) surface is only of "multiplicity 4" (i.e. 4 general symmetry equivalent positions) while the multiplicity of plane symmetry group p6mm is 12 , ref. [7]).

Note that considerations of the intersection of plane symmetry elements and of the relative strength of the deposit-substrate and inter-molecule interaction energies are not sufficient to predict the "apparent shape and mutual arrangement" of the $\mathrm{F}_{16} \mathrm{CoPc}$ molecules in a monolayer. For that one has to consider the nature of the "imaging signal" as well. Since STM images are in essence maps of the local density of electronic states, the quantum-theoretical geometry of isolated $\mathrm{F}_{16} \mathrm{CoPc}$ molecules does not need to be revealed in them.

From the viewpoint of the plane symmetry, the group $\mathrm{p} 4 \mathrm{~mm}$ may be realized for a metal phthalocyanine when two molecules (with point symmetry $4 \mathrm{~mm}$ ) "pair up", Fig. 1 right hand side, to form one motif of this plane group. Such an effect and plane group $\mathrm{p} 4 \mathrm{~mm}$ have actually been observed experimenttally for $\mathrm{SnPc}$ on $\mathrm{Ag}$ (111) [8]. Either identical or opposite sides of the individual molecules (that make up the pair) can thereby be in contact with the surface of the substrate. A "pairing up" of two molecules into one motif of the plane group $\mathrm{p} 4 \mathrm{~mm}$ is indicative of strongly anisotropic inter-molecule interactions and results in a larger unit cell than in plane group p4. Plane group $\mathrm{p} 4 \mathrm{~mm}$ may also be realized for metal phthalocyanine without "pairing up" of the molecules.
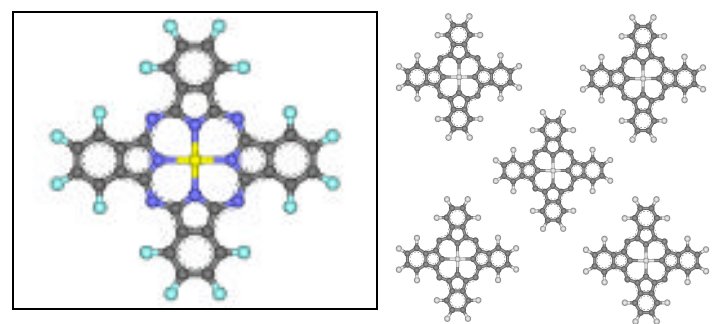

FIGURE 1. Model of the structure of fluorinated cobalt phthalocyanine $\left(\mathrm{F}_{16} \mathrm{CoPc}\right)$ (left) and one of the possible $2 \mathrm{D}$ arrangements of this molecule in plane group $\mathrm{p} 4 \mathrm{~mm}$ (right). The $2 \mathrm{D}$ point symmetry group of this molecule is $4 \mathrm{~mm}$. All other (homologous) phthalocyanines (where the central Co atom may be replaced by a transition-metal atom of valence two, all of the F atoms may be replaced by other halogens or hydrogen atoms) possess the same point symmetry. Some of these molecules are flat, e.g. $\mathrm{F}_{16} \mathrm{CoPc}$ or $\mathrm{CuPc}$. Others have their central metal atom out of plane, e.g. SnPc [8] or PbPc.

From the $2 \mathrm{D}$ point group of the $\mathrm{F}_{16} \mathrm{CoPc}$ molecule, it is unlikely that the 5 plane groups that contain glide lines in oblique and rectangular 2D-lattices and the plane group p4gm will be realized for its monolayers on graphite. The 5 plane groups with hexagonal 2D lattices are also unlikely as symmetries of an $\mathrm{F}_{16} \mathrm{CoPc}$ monolayer on substrates with the plane groups p6mm or $\mathrm{c} 2 \mathrm{~mm}$.

The quantification of the plane symmetry of a monolayer of $\mathrm{F}_{16} \mathrm{CoPc}$ on graphite with a popular $\mathrm{CIP}$ computer program [3], resulted in residual indices for the possible plane symmetries as given in Table 1. Plane group c2mm has been excluded from this table because the Fourier transform of the respective raw STM image, did not show systematic absences that were caused by the centering of a rectangular lattice [13].

TABLE 1. Amplitude and phase residuals for possible plane groups of a monolayer of $\mathrm{F}_{16} \mathrm{CoPc}$ on graphite.

\begin{tabular}{|l|l|l|l|l|l|l|}
\hline & p2 & p1m1 & p11m & p2mm & p4 & p4mm \\
\hline Fres in $\%$ & & 15.4 & 15.4 & 15.4 & 27.0 & 28.3 \\
\hline ares in $^{0}$ & 20.9 & 17.9 & 19.3 & 28.6 & 21.3 & 31.2 \\
\hline
\end{tabular}

Figure 2 shows raw STM data from a monolayer of $\mathrm{F}_{16} \mathrm{CoPc}$ on graphite as background and symmetry enforced versions of this data as insets. Because the signal in the z-direction is based on a quantum mechanical tunneling effect, STMs are much more sensitive in this direction than they are in any direction in the $x-y$ plane. The "enforcing" of plane symmetries is applied to this plane only so that the total tunneling signal per unit area is not affected. Enforcing plane symmetries on STM images results, therefore, only in lateral shifts of "symmetry averaged" tunneling signals.

In the absence of detailed free energy calculations, we conclude preliminarily from Table 1 and Fig. 2 that $\mathrm{p} 4$ or $\mathrm{p} 4 \mathrm{~mm}$ are the probable plane groups for the arrangement of a monolayer of $\mathrm{F}_{16} \mathrm{CoPc}$ on graphite (and that the interaction energy between deposit and substrate is not high). This conclusion is based on the fact that the phase residual is not much higher for $\mathrm{p} 4$ than for p2. Similarly, the phase residual is not much higher for $\mathrm{p} 4 \mathrm{~mm}$ than for $\mathrm{p} 2 \mathrm{~mm}$.

Note that p2 does not have an amplitude residual because $F(H, K)=F(-H,-K)$ is a property of the Fourier transform itself. From the difference in the phase residuals of the plane groups $\mathrm{p} 2 \mathrm{~mm}, \mathrm{p} 1 \mathrm{~m} 1$ and $\mathrm{p} 11 \mathrm{~m}$, one can conclude that the two mirror lines are not exactly perpendicular to each other.

For comparison, the equivalent of plane group p2 has been inferred by other authors from experimental STM data from a CoPc monolayer on an (111) oriented $\mathrm{Au}$ substrate [9]. There was, however, no quantification of this symmetry so that this result remains open to discussion. The qualitative symmetry inference of these authors may have been affected simply by artifacts of their microscope's calibration. 


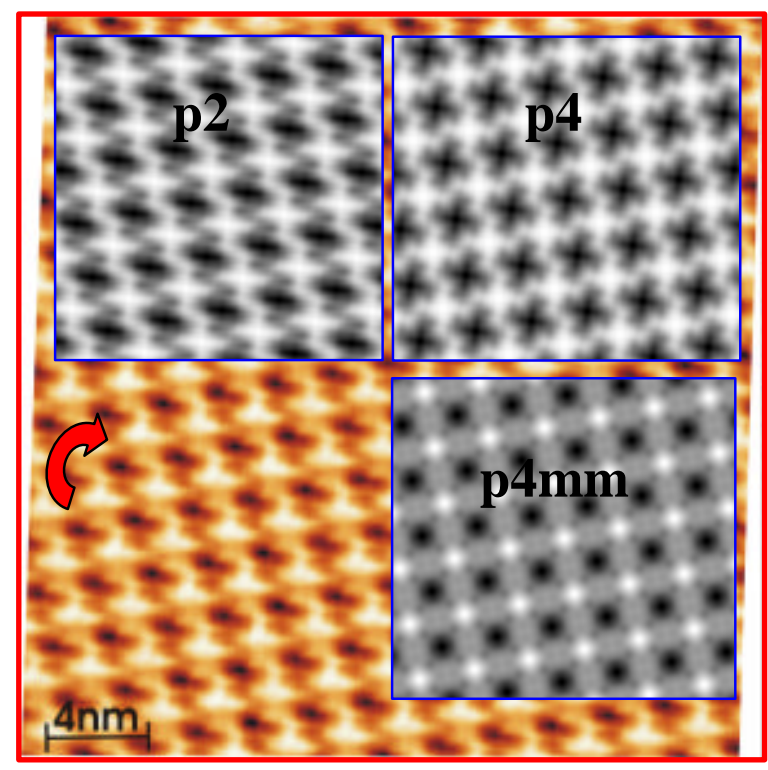

FIGURE 2. STM images of an $\mathrm{F}_{16} \mathrm{CoPc}$ monolayer on graphite, raw data as background and in $3^{\text {rd }}$ quadrant, symmetry enforced data with respective labels as insets. Note that compared to the "symmetrized" data, the raw data appear to be "clockwise twisted (see arrow), sheared, and anisotropically stretched". The motive of the p2 and p4 maps is rotated clockwise with respect to its counterpart in the $\mathrm{p} 4 \mathrm{~mm}$ map.

Figure 3 compares p4 enforced versions of STM data from two different $\mathrm{F}_{16} \mathrm{CoPc}$ monolayers on graphite substrate samples that were recorded with the same microscope but different tunneling tips. While the molecules in one of these images appear "rather clear", they seem to be somehow "washed out" in the other image. A possible explanation of these two different appearances is that the tunneling tip may have been for one sample close to the ideal single "atomically sharp tip", and for the other sample far from that ideal. (One well known example of such a non-ideal tip is a "blunt tip" that results from a multitude of atomically sharp tips all contributing to the same image and leading to rather complex convolutions.)

Figure 4 shows STM images of an $\mathrm{F}_{16} \mathrm{CoPc}$ monolayer on a (110) oriented surface of silver. CIP of the raw date gave in this case the plane symmetry group $\mathrm{p} 2$ with a phase residual of approximately $10^{\circ}$ (that varies slightly from area to area). The 2D lattice was identified as being oblique with an angle of about $72^{\circ}$ to $73^{\circ}$ and unit cell vector lengths that differ by approximately $10 \%$ from each other (again varying slightly from area to area). Note that one of the plane groups that were predicted for an $\mathrm{F}_{16} \mathrm{CoPc}$ monolayer on silver (110) from Curie's symmetry principle [12] has indeed been identified by CIP. Since this result is not unexpected, we take it as an assurance of the general utility of the CIP procedure for surface science studies.
It is also interesting to compare the STM signal counter map of the (noise filtered and) translation averaged ( $\mathrm{p} 1)$ version of the raw data from this sample with its (noise filtered and) p2 symmetry enforced counterpart, Fig. 5. The raw data of this STM image must have been of a high quality because the probesample interaction signal strength redistribution that results from the symmetry enforcement is rather small.

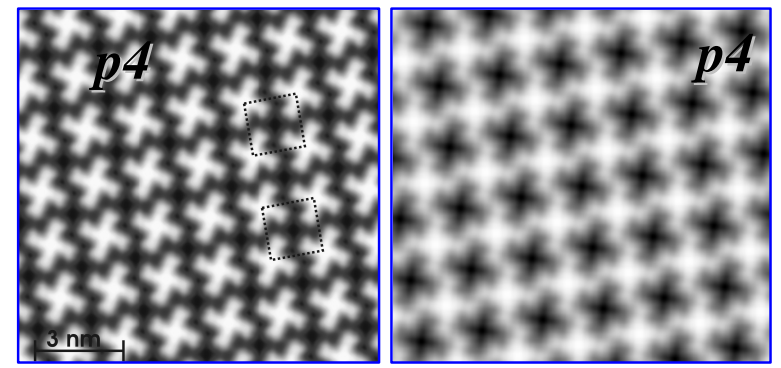

FIGURE 3. p4 symmetry enforced versions of STM data from two different samples of an $\mathrm{F}_{16} \mathrm{CoPc}$ monolayer on graphite, recorded with the same microscope but with two different tips. (Two square 2D unit cells of the 2D lattice are marked in the "rather clear" image. The right hand side data are also show as $1^{\text {st }}$ quadrant of Fig. 2.)

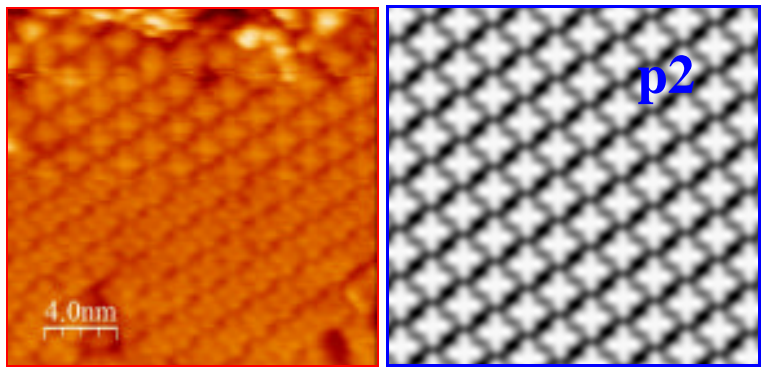

FIGURE 4. STM images of an $\mathrm{F}_{16} \mathrm{CoPc}$ monolayer on silver (110); (left) raw data, (right) noise filtered and p2 enforced data. Note that the raw data is quite noisy, but that the expected shape of the molecules is reasonably well revealed.
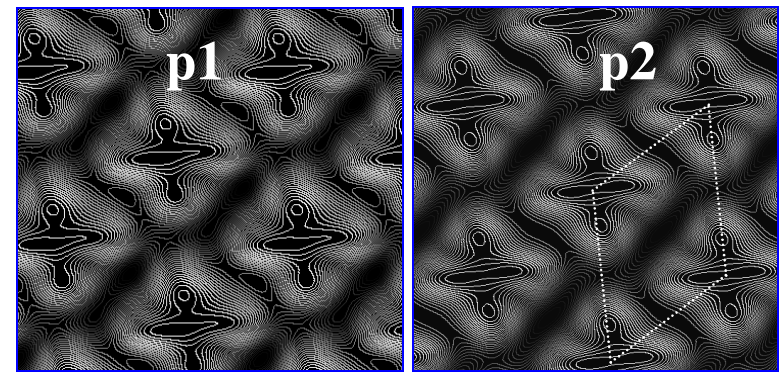

FIGURE 5. Counter maps of the STM data of fig. 4, an $\mathrm{F}_{16} \mathrm{CoPc}$ monolayer on silver (110); (left) plane group $\mathrm{p} 1$, resulting from translation averaging, (right) plane group $\mathrm{p} 2$ with sketch of 2D unit cell, resulting from the enforcement of two-fold axes at the center of the molecules. (Noise filtering was achieved by excluding very high spatial frequencies). 


\section{ASSESSING AND IMPROVING THE PERFORMANCE OF SCANNING PROBE MICROSCOPES}

The discussions above lead us to another potential usage of CIP of SPM images. Provided that a calibration standard with known periodic features, i.e. known plane group for the whole sample and known point group of the periodic motive, is available, the overall performance and the correctness of the calibration of the SPM can be straightforwardly assessed. In these cases, the amplitude and phase residuals provide quantitative measures of the overall performance and calibration of the microscope.

As long as the symmetry of the calibration standard is high, e.g. plane group p4mm (with multiplicity 8) or even better p6mm, the amplitude and phase residuals for all of their subgroups can also be used as quantitative measures for the existence of certain combinations of symmetry elements. The combined effects of all kinds of deviations from a "perfect SPM", e.g. an "effective" probe tip shape that deviates significantly from point symmetry group $\infty \mathrm{m}$, excessive noise, mis-calibrations of the $\mathrm{x}-\mathrm{y}$ step sizes, and non-linearity can, thus, be detected in the experimental SPM data.

Note that the whole procedure is quite similar to standard practices in HRTEM image-based electron crystallography $[5,6]$. For example, after the phase contrast transfer function of the electron microscope has been deconvoluted from the experimental image, the correct plane group of the projected electrostatic potential can be identified by its low phase and amplitude residuals. Note also that HRTEM studies of halogenated phthalocyanines revealed plane group p4mm [11] (in compliance with Curie's symmetry principle [12]). Imposing this plane symmetry removed the imperfections of the HRTEM imaging process from the structural data of the molecules [11].

\section{SUMMARY AND CONCLUSIONS}

Symmetry is an abstract mathematical concept and has been said to lie in the "eye of the beholder" for real world objects such as 2D-images of probe-sample interactions that were recorded with a SPM. Symmetry can, however, be quantified by crystallographic image processing. These quantifications can be used to assess the overall performance and correctness of the calibration of all kinds of SPMs. Enforcing the most probable plane symmetry on a SPM image results in the removal of artifacts that are due to the microscopical imaging process. Plane symmetry enforcing on a SPM image may also be considered as being equivalent to scanning probe tip "symmetrization" into an "ideal needle".
Since corrections for "sample tilt" are being performed quite regularly in scanning probe microscopy on the basis of presumed prior knowledge about the probable strength of the sample-probe interaction signal at the margins of a sample area, one may with even more confidence enforce quantified probable plane symmetries on the raw SPM data in order to remove similarly obvious imperfections of the imaging process from the experimental surface science data.

\section{ACKNOWLEDGMENTS}

This research was supported by awards from the Oregon Nanoscience and Microtechnologies Institute. Additional support from Portland State University's Venture Development Fund is acknowledged.

\section{REFERENCES}

1. P. Moeck, "Nanometrology device standards for scanning probe microscopes and processes for their fabrication and usage", US Patent No. 7,472,576.

2. J. S. Villarrubia, "Algorithms for Scanned Probe Microscope Image Simulation, Surface Reconstruction, and Tip Estimation, J. Res. Natl. Inst. Stand. Technol. 102 (1997) 425-454.

3. Hovmöller, S.: "CRISP: crystallographic image processing on a personal computer", Ultramicroscopy 41 (1992) 121-135.

4. M. J. Landsberg and B. Hankamer, "Symmetry: A guide to its applications in 2D electron crystallography”, J. Struct. Biolog. 160 (2007) 332-343.

5. X. Zou and S. Hovmöller, "Electron crystallography: imaging and single-crystal diffraction from powders", Acta Cryst. A 64 (2008) 149-160; open access:

http://journals.iucr.org/a/issues/2008/01/00/issconts.html 6. X. D. Zou and S. Hovmöller, "Electron Crystallography: Structure Determination by HREM and Electron Diffraction", in: Industrial Applications of Electron Microscopy (Ed. Z. R. Li), p. 583-614, Marcel Dekker Inc., 2003.

7. T. Hahn (Ed.), "Brief Teaching Edition of Volume A, Spacegroup symmetry", International Tables for Crystallography, $5^{\text {th }}$ revised edition, IUCr, Chester 2005.

8. M. Lackinger, and M. Hietschold, "Determining adsorption geometry of individual tin-phthalocyanine molecules on $\mathrm{Ag}(111)$ - a STM study at submonolayer coverage", Surface Science $\mathbf{5 2 0}$ (2002) L619-L624.

9. M. Takada and H. Tada, "Low temperature scanning tunneling microscopy of phthalocyanine multilayers on Au (111) surfaces", Chem. Phys. Lett. 392 (2004) 265-269.

10. C. Ludwig, R. Strohmaier, J. Peterson, B. Gompf, and W. Eisenmenger, "Epitaxy and scanning tunneling microscopy image contrast of copper-phthalocyanine on graphite and $\mathrm{MoS}_{2}$ ", J. Vac. Sci. Technol. B 12 (1994) 1963-1966.

11. D. J. Smith, J. R. Fryer, and R. A. Camps, "Radiation damage and structural studies: Halogenated Phthalocyanines", Ultramicroscopy 19 (1986) 270-298.

12. P. Curie, "Sur la symétrie dans les phénomènes physiques, symétrie d'un champ électrique et d'un champ magnétique", $J$. de Physique 3 (1894) 393-415.

13. P. Moeck, B. Moon Jr., M. Abdel-Hafiez, and M. Hietschold, "Quantifying and enforcing the two-dimensional symmetry of scanning probe microscopy images of periodic objects", Proc. NSTI 2009, Houston, May 3-7, 2009, Vol. I (2009) 314-317, (ISBN: 978-1-4398-1782-7). 